\title{
Flow Cytometry Baseline on Peripheral Leukocyte Cell Profiles for Dairy Goat Kids
}

\author{
${ }^{1}$ Darcy M. Fletcher, ${ }^{1}$ Megan B. Vogt, ${ }^{1}$ Hannah E. Pirner, ${ }^{2}$ Ann M. Hess, \\ ${ }^{3}$ Richard A. Bowen, ${ }^{1}$ Marcela Henao-Tamayo and ${ }^{1}$ Torsten M. Eckstein
${ }^{1}$ Department of Microbiology, Immunology and Pathology, College of Veterinary Medicine and Biomedical Sciences, Campus Delivery 1682, Colorado State University, Fort Collins, CO 80523, USA
${ }^{2}$ Department of Statistics, College of Natural Sciences, Campus Delivery 1877, Colorado State University, Fort Collins, CO 80523, USA
${ }^{3}$ Department of Biomedical Sciences, College of Veterinary Medicine and Biomedical Sciences, Campus Delivery 1683, Colorado State University, Fort Collins, CO 80523, USA

Received 2013-08-21, Revised 2013-09-09; Accepted 2013-09-26

\begin{abstract}
Small ruminants, like goats, would make excellent animal models for not only infectious diseases in large ruminants but also analogous diseases in humans, such as human tuberculosis, Crohn's disease, melioidosis and brucellosis. The main disadvantage for the small ruminant model is the lack of sufficient baseline data on normal, healthy goat kids. Furthermore, most reagents (antibodies and the like) were not developed for goats or sheep. It is important to demonstrate that available resources, especially from the bovine system, cross-react with the caprine and/or ovine system. Finally, potential breed differences have to be evaluated before goat or sheep studies are compared. In this study, leukocyte cell populations were defined in twenty-six dairy goat kids via flow cytometry. We report no significant differences between three breeds of dairy goat kids and demonstrate the effective use of various antibodies for caprine immune cell markers. No breed-specific differences were detected for any leukocyte cell population or for markers specific for various antigen-presenting cells or T cell populations. Interestingly, however, statistical significant differences were found for leukocyte cell populations for the two different time points two weeks apart presented in this study.
\end{abstract}

Keywords: Goat, Peripheral Blood Leukocytes, Cell Surface Marker, Flow Cytometry

\section{INTRODUCTION}

Animal models are key elements in evaluating pathogenicity and virulence of various bacterial and viral agents (Horvat, 2009; Ross et al., 2012; Hibiya et al., 2011; Munson et al., 2012; McConnell et al., 2013; Uzal and McClane, 2012). While the mouse model is a sufficient animal model for most human infectious diseases (Yi and Li, 2012; Hviid et al., 2010; Williams et al., 2009; Drescher and Sosnowska, 2008; Groschup and Buschmann, 2008; Zhang and Moss, 2012; Shi et al., 2011), increasing attempts are being made to use animal models more applicable to zoonotic and animal diseases (Kahn, 2012). The mouse model has distinct advantages over other animal models (financial restrictions, availability of various reagents, presence of various mouse strains), but, in the case of infectious diseases, often lacks correlation to the original host and its immune responses. This correlation is even more important for chronic and recurrent infectious diseases present in humans and animals such as tuberculosis, melioidosis and brucellosis (Padilla-Carlin et al., 2008; Dharmadhikari and Nardell, 2008; Kahl-McDonagh et al., 2006; Soffler et al., 2012).

\section{Corresponding Author: Torsten M. Eckstein, Department of Microbiology, Immunology and Pathology, College of Veterinary Medicine and Biomedical Sciences, Campus Delivery 1682, Colorado State University, Fort Collins,} CO 80523, USA Tel: (970) 491-2015 Fax: (970) 491-1815 
Considering the amount of financial, space and material resources expended on large animals (horses, cattle), goats serve as excellent animal models for infectious diseases found in ruminants and humans. Over the last decade many reagents were developed for use in ruminants. Although they were designed primarily for use in cattle, most of them exhibit crossreactivity with closely related ruminants, such as sheep and goat (Mosaad et al., 2006).

Unlike with mouse strains, individual goats do not always react in a manner similar to each other. Furthermore, goats were bred for different purposes, such as fiber, meat and dairy. Many different breeds exist within these groups and it is not known yet how their immune system is organized and might react to certain pathogens. It is also not known if data from various goat breeds can easily be compared or if their healthy immune systems fundamentally differ. Although many studies focus on the immune system of goats, only a couple of publications studied the cellular immune system in goat kids a few weeks old. Neither publication focused on potential breed differences but instead detailed new techniques to characterize various cell populations. With the trend of using small ruminants as key animal models for ruminant infectious diseases, it is important to discover and evaluate any potential breed differences to allow for better breed selection for animal studies and comparison of various studies and their conclusions using different breeds. Recently, flow cytometry has played a stronger role in describing various cell populations of animals in models other than the mouse model.

Here, we provide the flow cytometry baseline results on dairy goat kids for various leukocyte cell populations as well as baseline data on several $\mathrm{T}$ cell populations and antigen-presenting cell markers.

\section{MATERIALS AND METHODS}

\subsection{Animals}

Twenty-six goat kids aged two to five days were purchased from CCI/Juniper Valley Products (Canon City, Colorado) and transferred the same day to our campus. The goat kids were housed on Colorado State University Foothills Campus in accordance with CSU animal ethics regulations (\#11-3120A). All goats were housed in the same barn until the age of seven weeks. Goats were fully milk fed (three times a day) with whole cow milk purchased from Walmart. The goats were less than 6 weeks old at the time of the blood draw. By breed, there were fourteen Alpines, three Saanens, seven
Anglo-Nubians, one LaMancha and one Toggenburg. The LaMancha and Toggenburg were not considered in the comparative analyses because there is only a single goat for those breeds; however, data obtained for the two animals will still be presented. The fourteen Alpines were further divided into the following sub-breeds: Alpine-Chamoise (seven goats), Alpine-Cou Blanc (four goats), Alpine-Sundgau (two goats) and Alpine Broken (one goat). The three Saanens were identified as two Saanen-mix and one Saanen-Sable. The Anglo-Nubian breed was not further divided.

\subsection{Blood Draw and White Blood Cell Preparation}

Two blood draws were performed due to the young age of the goat kids and the amount of blood needed to perform all anticipated cell marker-staining experiments. The first blood draw was performed at age 3.5 weeks, while the second blood draw was performed at age 5.5 weeks. For each blood draw four milliliters of blood were obtained aseptically from the jugular vein into an EDTA solution for immediate analyses. Each sample was transferred to a separate $50 \mathrm{~mL}$ polypropylene conical tube. Samples were treated with Gey's solution $\left(155 \mathrm{mM} \mathrm{NH} 4 \mathrm{Cl}, 1 \mathrm{mM} \mathrm{KHCO}_{3}\right)$ to lyse the red blood cells. A volume of Gey's solution equal to that of the blood was addedto each sample. After five minutes incubation, Phosphate Buffered Saline (PBS) was added in equal volume to Gey's solution. White blood cells were pelleted via centrifugation for $5 \mathrm{~min}$ at $259 \mathrm{~g}$ at $4^{\circ} \mathrm{C}$. The supernatant was discarded and pellets washed twice in $10 \mathrm{~mL}$ PBS each, centrifuging and discarding the supernatant of each wash. After the second wash, clean pellets were resuspended in complemented RPMI media (RPMI media, $8.7 \%$ of Fetal Bovine Serum, 1M HEPES Buffer, 50× MEM without L-glutamine, $100 \mathrm{mM}$ Sodium Pyruvate, $200 \mathrm{mM}$ L-glutamine solution, Penicillin $\left(10,000\right.$ units $\left.\mathrm{mL}^{-1}\right) /$ Streptomycin $(10,000$ ug $\mathrm{mL}^{-1}$ ) solution) (cRPMI). Pellets containing a visible red blood cell layer were treated with an additional $5 \mathrm{~mL}$ Gey's solution for 1-2 min before adding $10 \mathrm{~mL}$ PBS. Pellets were reformed via centrifugation under the aforementioned conditions and then resuspended in cRPMI media.

\subsection{Antibodies for Cell Markers}

Conjugated antibodies for flow cytometry cell surface marker staining were used for the following cell markers: CD1, CD4, CD8, CD335, MHC class II, WC1 and CD14 (Table 1). 
Table 1. Conjugated monoclonal antibodies for various cell surface markers used in this study, their primary reactivity, isotype, fluorophore and the cell phenotypes they primarily represent

\begin{tabular}{|c|c|c|c|c|c|c|c|}
\hline $\mathrm{mAb}$ & Reactivity & Clone & Iso-type & Fluoro-phore & Cell phenotype & Company & cat.No \\
\hline$\overline{\mathrm{CD} 1}$ & ovine & 20.27 & IgG1 & A647 & Antigen Presenting (lipids) & AbD Serotec & MCA2212A647 \\
\hline CD4 & Ovine & 44.38 & $\operatorname{IgG} 2 \mathrm{a}$ & A647 & T Helper Lymphocyte & AbD Serotec & MCA2213A647 \\
\hline $\mathrm{CD} 8$ & Bovine & CC63 & $\operatorname{IgG} 2 \mathrm{a}$ & FITC & Cytotoxic T Lymphocyte & AbD Serotec & MCA 837F \\
\hline CD335 & Bovine & AKS1 & IgG1 & A488 & Natural Killer cells & AbD Serotec & MCA 2365A488 \\
\hline MHCII & Ovine & 34.68 & $\operatorname{IgG} 2 \mathrm{a}$ & FITC & Antigen Presenting (lipids) & AbD Serotec & MCA2226F \\
\hline WC1 & Bovine & $\mathrm{CC} 15$ & $\operatorname{IgG} 2 \mathrm{a}$ & FITC & $\gamma \delta$ T Lymphocyte & AbD Serotec & MCA $838 \mathrm{~F}$ \\
\hline CD14 & Human & M532 & $\operatorname{IgG} 2 \mathrm{a}$ & $\mathrm{PE}$ & Monocyte & BioLegend & 301806 \\
\hline
\end{tabular}

\subsection{Staining of Cell Markers}

Whole lysed blood was stained with monoclonal antibodies to identify cell surface markers. Cells $\left(5 \times 10^{6}\right.$ cells $\mathrm{mL}^{-1}$ ) were incubated for $20 \mathrm{~min}$ at $4^{\circ} \mathrm{C}$ with $10 \mu \mathrm{L}$ of each monoclonal antibody. Following the incubation, the cells were washed three times with $200 \mu \mathrm{L}$ PBS, centrifuged at $260 \mathrm{~g}$ and resuspended in $200 \mu \mathrm{L}$ PBS.

\subsection{Flow Cytometry}

Samples were analyzed via a flow cytometer (FACSCantoII, Becton Dickinson, USA) equipped with BD FACSDiva software (Becton Dickinson, USA). A minimum of 1500 events was collected per sample. Profiles were analyzed with FlowJo (FlowJo, USA). Four panels were analyzed: CD4/CD8, CD4/WC1, CD1/CD14 and MHCII/CD14. Panels CD4/CD8 and CD4/WC1 were performed on the first blood draw at the age of 3.5 weeks, while CD14/CD1 and CD14/MHC class II were measured on the second blood draw at the age of 5.5 weeks. Originally, CD335 was also included. Since no measurable amounts of CD335 positive cells were detected, this data set was not included in the overall analyses.

The fluorescence in each sample was determined using a BD FACSCanto II (BD Bioscience) and the acquired data was analyzed using the FlowJo software (TreeStar, Ashland, OR). Gate strategy included selection of cell populations according to their side/forward scatter profile. Thereafter, the fluorescence of positive cells was compared to their corresponding isotype-matched control and the percentage of positive cells and intensity of fluorescence was recorded as percentage of positive cells and Mean Fluorescence Channel (MFC), respectively.

The gating strategy to identify granulocytes, monocytes and lymphocytes is presented in each figure. Briefly, Forward Scatter (FSC-A) represents the cell size in a linear pattern, while the Side Scatter (SSC-A) represents the granulation of each cell in a linear pattern. Granulocytes have strong granulation with a variable cell size, while lymphocytes and monocytes have less granulation. Lymphocytes are smaller in size than monocytes. Cell population according to their size and granulation are encircled. The individual gating procedure for each panel is described in each figure specifically. Some cell marker analyses were also backgated to detect the location of the positive cells within the original plot. When analyzing cells for the marker CD14, positive cells were back gated to identify the cell type they originated from. The back gating approach resulted in two CD14+ cell populations: one associated with monocytes and one associate with granulocytes. 2.6. Statistical analyses.

Statistical analysis was done using SAS 9.3 (SAS, Cary, NC). For the antibody data, one-way ANOVA Ftests were done to test for differences between breeds for each antibody separately. A Benjamini-Hochberg adjustment was used to account for multiple testing (Benjamini and Hochberg, 1995). For the granulocyte, lymphocyte and monocyte data, one-way ANOVA Ftests were done to test for differences between breeds for each week and draw separately. A BenjaminiHochberg adjustment was again used to account for multiple testing (Benjamini and Hochberg, 1995). In addition, paired t-tests were done to test for differences between panels and time points.

\section{RESULTS}

\subsection{Gating Results at the Age of 3.5 and 5.5 Weeks}

The goat whole lysed blood cell samples were analyzed via flow cytometry according to their SSC-A and FSC-A profiles to determine presence and abundance of the three major cell populations: Lymphocytes, monocytes and granulocytes. Accordingly, granulocytes (variable sizes, high granulation) were identified as SSCAhigh/FSCAvariable whereas lymphocytes (small size, low granulation) were identified as SSC-A ${ }^{\text {low }} / \mathrm{FSC}-\mathrm{A}^{\text {low }}$ and monocytes (medium size, low granulation) as SSC$\mathrm{A}^{\text {low }} /$ FSC-A ${ }^{\text {medium }}$ (Fig. 1). 


\begin{tabular}{|c|c|c|c|c|c|c|c|}
\hline & Lymp & phocytes & Granulo & K... & mphocytes & Granulo & onocytes \\
\hline \multirow[t]{2}{*}{ Tag \# } & \multirow[t]{2}{*}{ Breed } & \multicolumn{3}{|c|}{3.5 weeks } & \multicolumn{3}{|c|}{5.5 weeks } \\
\hline & & $\begin{array}{l}\text { Lympho- } \\
\text { cytes }\end{array}$ & $\begin{array}{l}\text { Mono- } \\
\text { cytes }\end{array}$ & $\begin{array}{l}\text { Granulo- } \\
\text { cytes }\end{array}$ & $\begin{array}{l}\text { Lympho- } \\
\text { cytes }\end{array}$ & $\begin{array}{l}\text { Mono- } \\
\text { cytes }\end{array}$ & $\begin{array}{l}\text { Granulo- } \\
\text { cytes }\end{array}$ \\
\hline 6578 & Alpine & 46.4 & 3.38 & 12.8 & 44.9 & 2.88 & 11 \\
\hline 6583 & Alpine & 42.2 & 2.31 & 8.6 & 60 & 7.59 & 10.2 \\
\hline 6593 & Alpine & 39.9 & 2.76 & 19 & 53.6 & 5.97 & 16.7 \\
\hline 6617 & Alpine & 46.6 & 5.49 & 23.2 & 41.4 & 2.83 & 6.1 \\
\hline 6618 & Alpine & 20.9 & 0.87 & 12.4 & 60.6 & 5.07 & 7.45 \\
\hline 6621 & Alpine & 49.6 & 5.43 & 7.69 & 55.1 & 4.26 & 15.6 \\
\hline 6629 & Alpine & 56.2 & 5.09 & 14.7 & 40.7 & 2.17 & 9.38 \\
\hline 6630 & Alpine & 52.7 & 2.93 & 7.9 & 30.3 & 2.09 & 3.65 \\
\hline 6631 & Alpine & 49.7 & 5.02 & 10.9 & 51.5 & 5.12 & 16.3 \\
\hline 6632 & Alpine & 55.7 & 6.63 & 12.3 & 64.7 & 2.77 & 5.23 \\
\hline 6670 & Alpine & 27.1 & 2.95 & 26.4 & 52.8 & 2.95 & 4.97 \\
\hline 6673 & Alpine & 61.2 & 8.38 & 9.79 & 49.5 & 2.29 & 5.91 \\
\hline 6677 & Alpine & 64.3 & 4.34 & 7.76 & 53.2 & 3.05 & 2.86 \\
\hline 6678 & Alpine & 44.4 & 3.61 & 17.8 & 63.4 & 3.47 & 9.06 \\
\hline Average & Alpine & 46.921 & 4.228 & 13.66 & 51.55 & 3.751 & 8.886 \\
\hline 6616 & Anglo-Nubian & 51.8 & 2.3 & 8.94 & 17 & 0.78 & 3.61 \\
\hline 6622 & Anglo-Nubian & 39.3 & 2.38 & 13.5 & 41.2 & 4.12 & 15 \\
\hline 6623 & Anglo-Nubian & 45.1 & 2.37 & 14 & 31.8 & 2.56 & 23.9 \\
\hline 6625 & Anglo-Nubian & 60.2 & 4.15 & 9.03 & 36.3 & 3.22 & 16 \\
\hline 6679 & Anglo-Nubian & 15 & 1.49 & 33.1 & 52.6 & 0.67 & 3.62 \\
\hline 6682 & Anglo-Nubian & 53.8 & 3.67 & 5.82 & 62 & 1.84 & 6.51 \\
\hline 6686 & Anglo-Nubian & 40.4 & 2.75 & 21.8 & 36.4 & 2.23 & 11.6 \\
\hline Average & Anglo-Nubian & 43.657 & 2.73 & 15.17 & 39.61 & 2.203 & 11.46 \\
\hline 6620 & Saanen & 59.3 & 2.87 & 14.7 & 50.5 & 3.76 & 9.25 \\
\hline 6624 & Saanen & 33.4 & 2.5 & 24.3 & 9.59 & 0.53 & 0.53 \\
\hline 6671 & Saanen & 32.3 & 2.58 & 27 & 56.3 & 3.97 & 10.6 \\
\hline Average & Saanen & 41.667 & 2.65 & 22.00 & 38.797 & 2.753 & 6.79 \\
\hline 6619 & La Mancha & 36.5 & 1.94 & 7.4 & 32.9 & 4.64 & 19.1 \\
\hline 6627 & Toggenburg & 59.7 & 4.03 & 5.21 & 35.6 & 5.53 & 24.6 \\
\hline
\end{tabular}

Fig. 1. Flow cytometry plots of goat $\# 6593$ (as one example for the gating strategy) at age 3.5 weeks (left plot) and at the age of 5.5 weeks (right plot). The two plots demonstrate the strategy of gating for all goats included in this study. Forward scatter represents the cell size in an exponential pattern, while side scatter represents the granulation of each cell in a linear pattern. Granulocytes have strong granulation, while lymphocytes and monocytes have less granulation. Lymphocytes are smaller in size than monocytes. Cell population according to their size and granulation are encircled. The table below the plots shows the amounts of lymphocytes, monocytes and granulocytes obtained at each time points as well as the averages for each breed

Figure 1 presents the data obtained within the second set (panel CD4/CD8) of week 3.5 in comparison with results obtained in week 5.5 of age. Statistical analyses of the various breeds at each time point and panel did not reveal any significant breed-associated differences (Table 2).

\subsection{Gating Results for Various Lymphocyte Populations}

Lymphocyte cell populations were gated according to their SSC-A ${ }^{\text {low }} /$ FSC-A ${ }^{\text {low }}$ profiles (Fig. 2 and 3). Five major lymphocyte populations were analyzed: Cells with positive fluorescence for $\mathrm{mAb}$ recognizing $\mathrm{CD} 4$ (CD4+ T cells), CD8 (CD8+ $\mathrm{T}$ cells), CD4/CD8 double positive $\mathrm{T}$ cells, CD4/CD8 double negative $\mathrm{T}$ cells and $\mathrm{WC} 1$ positive $\mathrm{T}$ cells $(\gamma \delta \mathrm{T}$ cells). CD335 was used as a marker for NKT cells, but only trace amounts were present and thus were not included in this analysis. Data for cells with positive surface expression for CD4 and/or CD8 are shown in Fig. 2, while those for WC1 and/or CD4 are shown in Fig. 3. 
Darcy M. Fletcher et al. / American Journal of Animal and Veterinary Sciences 8 (4): 177-189, 2013

Table 2. Statistical analyses of potential breed differences of average cell amounts of peripheral white blood cell populations for the two different time points and two different panels at week 3 (F: F test statistic for oneway ANOVA, BH p-value represents Benjamini-Hochberg adjusted p-value)

\begin{tabular}{llrlrlll}
\hline Cell type & Time point & Alpine & Anglo-Nubian & Saanen & F & P-value & BH p-value \\
\hline Granulovytes & Week 3 & 13.6600 & 15.1700 & 22.0000 & 1.681 & 0.210 & 0.437 \\
Granulovytes & Week 5 & 8.8860 & 11.4600 & 6.7900 & 0.838 & 0.446 & 0.613 \\
Lymphocytes & Week 3 & 46.9210 & 43.6570 & 41.6670 & 0.276 & 0.762 & 0.762 \\
Lymphocytes & Week 5 & 51.5500 & 39.6100 & 38.7970 & 2.414 & 0.114 & 0.344 \\
Monocytes & Week 3 & 4.2280 & 2.7300 & 2.6500 & 2.634 & 0.095 & 0.344 \\
Monocytes & Week 5 & 3.7510 & 2.2030 & 2.7530 & 2.404 & 0.115 & 0.344 \\
\hline
\end{tabular}
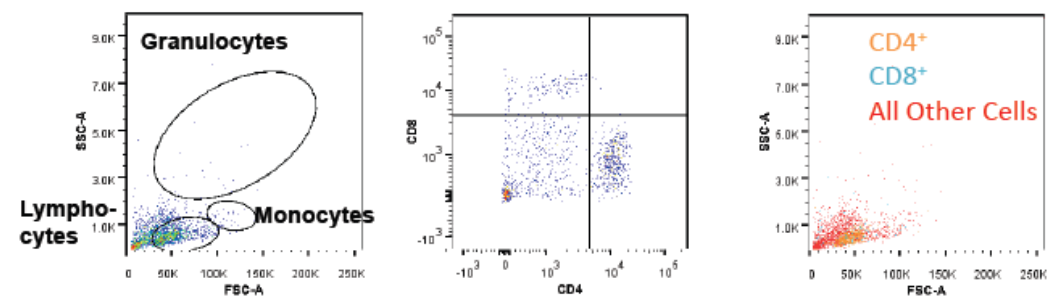

\begin{tabular}{|c|c|c|c|c|c|}
\hline Tag \# & Breed & CD4+ & CD8+ & CD4+/CD8+ & CD4-/CD8- \\
\hline 6578 & Alpine & 39.9 & 11.6 & 0.49 & 48 \\
\hline 6583 & Alpine & 36.9 & 13 & 0.39 & 49.8 \\
\hline 6593 & Alpine & 50.1 & 17.9 & 0.4 & 31.7 \\
\hline 6617 & Alpine & 41.3 & 15.6 & 0.65 & 42.5 \\
\hline 6618 & Alpine & 40.4 & 13.5 & 0 & 46.2 \\
\hline 6621 & Alpine & 37.8 & 6.08 & 0.1 & 56 \\
\hline 6629 & Alpine & 59.9 & 14.3 & 0.47 & 25.4 \\
\hline 6630 & Alpine & 43.5 & 16.3 & 0.25 & 39.9 \\
\hline 6631 & Alpine & 50.6 & 12.6 & 0.73 & 36 \\
\hline 6632 & Alpine & 42.8 & 17.8 & 0.64 & 38.7 \\
\hline 6670 & Alpine & 22.2 & 5.61 & 0.35 & 71.9 \\
\hline 6673 & Alpine & 44.9 & 14.5 & 0.47 & 40.1 \\
\hline 6677 & Alpine & 44.8 & 16.9 & 0.89 & 37.5 \\
\hline 6678 & Alpine & 52 & 19.2 & 0.51 & 28.3 \\
\hline Average & Alpine & 43.364 & 13.921 & 0.453 & 42.286 \\
\hline 6616 & Anglo-Nubian & 35.5 & 22.9 & 0.39 & 41.2 \\
\hline 6622 & Anglo-Nubian & 32.1 & 13.6 & 0.097 & 54.2 \\
\hline 6623 & Anglo-Nubian & 39.2 & 10.6 & 0.93 & 49.3 \\
\hline 6625 & Anglo-Nubian & 53.6 & 14.6 & 0.66 & 31.1 \\
\hline 6679 & Anglo-Nubian & 45.2 & 8.31 & 0.47 & 46 \\
\hline 6682 & Anglo-Nubian & 40.1 & 10.2 & 0.92 & 48.8 \\
\hline 6686 & Anglo-Nubian & 18.5 & 8.81 & 0 & 72.7 \\
\hline Average & Anglo-Nubian & 37.743 & 12.717 & 0.495 & 49.043 \\
\hline 6620 & Saanen & 53.4 & 14.7 & 1.03 & 30.9 \\
\hline 6624 & Saanen & 34.1 & 17 & 0.46 & 48.4 \\
\hline 6671 & Saanen & 42.5 & 7.58 & 0.18 & 49.7 \\
\hline Average & Saanen & 43.333 & 13.093 & 0.556 & 43.00 \\
\hline 6619 & LaMancha & 44.9 & 13.6 & 0 & 41.5 \\
\hline 6627 & Toggenburg & 48.7 & 12.9 & 0.41 & 38 \\
\hline
\end{tabular}

Fig. 2. Flow cytometry plots of goat \#6593 (as one example for the gating strategy) at age 3.5 weeks (second set). Left plot represents cell sorting according to cell size (FSC-A) and granulation (SSC-A). Lymphocytes are encircled as cells small in size and low in granulation. This cell population was used for analysis for markers for CD4 (x-axis) and CD8 (y-axis) (middle plot). Cells were then back-gated to the original FSC-A/SSC-A plot to identify the correct population of lymphocytes (plot at the right). The table below the flow cytometry plots contains data for the various cell subpopulations associated with the two markers for each goat as well as the average of each breed 

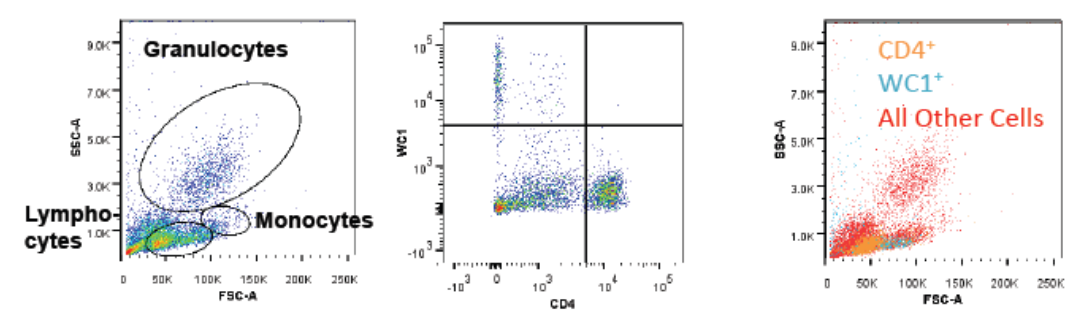

\begin{tabular}{|c|c|c|c|c|c|}
\hline & \multicolumn{2}{|c|}{$\mathrm{cos}$} & \\
\hline Tag \# & Breed & CD4+ & wc1+ & CD4+/WC1+ & CD4-MC1- \\
\hline 6578 & Alpine & 33.7 & 9.62 & 0.043 & 56.7 \\
\hline 6583 & Alpine & 28 & 25.6 & 0.19 & 46.2 \\
\hline 6593 & Alpine & 40.4 & 6.91 & 0.14 & 52.5 \\
\hline 6617 & Alpine & 29.9 & 27.1 & 0.13 & 42.8 \\
\hline 6618 & Alpine & 31.8 & 10.7 & 0 & 57.6 \\
\hline 6621 & Alpine & 31.8 & 16.4 & 0.15 & 51.7 \\
\hline 6629 & Alpine & 51.7 & 13.1 & 0.089 & 35.1 \\
\hline 6630 & Alpine & 34.1 & 26.8 & 0.17 & 38.9 \\
\hline 6631 & Alpine & 43.8 & 8.07 & 0.08 & 48 \\
\hline 6632 & Alpine & 35.9 & 21.7 & 0.11 & 42.3 \\
\hline 6670 & Alpine & 19.3 & 33 & 0 & 47.7 \\
\hline 6673 & Alpine & 36.1 & 24.2 & 0.082 & 39.6 \\
\hline 6677 & Alpine & 42 & 18 & 0.093 & 39.9 \\
\hline 6678 & Alpine & 31 & 21.8 & 0.21 & 47 \\
\hline Average & Alpine & 34.964 & 18.786 & 0.106 & 46.143 \\
\hline 6616 & Anglo-Nubian & 31.2 & 14.4 & 0.11 & 54.3 \\
\hline 6622 & Anglo-Nubian & 29.4 & 17.2 & 0.36 & 53 \\
\hline 6623 & Anglo-Nubian & 33.2 & 13.1 & 0.11 & 53.5 \\
\hline 6625 & Anglo-Nubian & 47.1 & 9.98 & 0.22 & 42.7 \\
\hline 6679 & Anglo-Nubian & 13.3 & 15.5 & 0 & 71.1 \\
\hline 6682 & Anglo-Nubian & 30.3 & 29.9 & 0.17 & 39.7 \\
\hline 6686 & Anglo-Nubian & 33 & 32.6 & 0.12 & 34.2 \\
\hline Average & Anglo-Nubian & 31.071 & 18.954 & 0.156 & 49.786 \\
\hline 6620 & Saanen & 45.5 & 15 & 0.15 & 39.3 \\
\hline 6624 & Saanen & 31.1 & 12.1 & 0.09 & 56.7 \\
\hline 6671 & Saanen & 35 & 17.3 & 0.19 & 47.5 \\
\hline Average & Saanen & 37.2 & 14.8 & 0.143 & 47.833 \\
\hline 6619 & LaMancha & 37.9 & 14.9 & 0.27 & 46.9 \\
\hline 6627 & Toggenburg & 40.5 & 17.9 & 0.11 & 41.4 \\
\hline
\end{tabular}

Fig. 3. Flow cytometry plots of goat \#6593 (as one example for the gating strategy) at age 3.5 weeks. Left plot represents cell sorting according to cell size (FSC-A) and granulation (SSC-A). Lymphocytes are encircled as cells small in size and low in granulation. This cell population was used for analysis for markers for CD4 (x-axis) and WC1 (y-axis) (middle plot). Cells were then back-gated to the original FSC-A/SSC-A plot to identify the correct population of lymphocytes (plot at the right). The table below the flow cytometry plots contains data for the various cell subpopulations associated with the two markers for each goat as well as the average of each breed

No statistical significant differences between the three breeds were found for any $\mathrm{T}$ cell population (Table 3 ).

\subsection{Gating Results for CD14 Bearing Cells and Antigen-Presenting Cells}

Whole lysed blood cell samples obtained from each goat at 5.5 weeks of age were used to identify cells with the CD14 marker (monocytes, granulocytes) in combination with molecules necessary for antigen presentation (CD1, MHC class II). While most of these cells should be monocytes, a significant portion was identified to be within the granulocyte population (Fig. 4). Interestingly, while all granulocytes bear CD14, only a portion of the monocytes bears this marker (32 to 92\%). No statistical significances for breed differences were detected (Table 4). MHC class II and CD1 molecules are antigen presenting cell surface molecules present on antigen-presenting cells (macrophages, dendritic cells). 

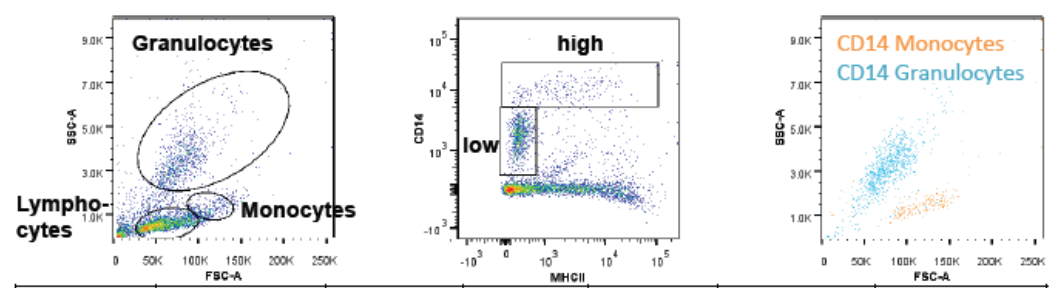

\begin{tabular}{|c|c|c|c|c|c|c|}
\hline Tag \# & Breed & $\begin{array}{l}\text { Lympho- } \\
\text { cytes }\end{array}$ & $\begin{array}{l}\text { Mono- } \\
\text { cytes }\end{array}$ & $\begin{array}{l}\text { Granulo- } \\
\text { cytes }\end{array}$ & $\begin{array}{l}\text { CD14 within } \\
\text { monocytes }\end{array}$ & $\begin{array}{l}\text { CD14 within } \\
\text { granulocytes }\end{array}$ \\
\hline 6578 & Alpine & 44.9 & 2.88 & 11 & 2 & 11.2 \\
\hline 6583 & Alpine & 60 & 7.59 & 10.2 & 2.42 & 10.4 \\
\hline 6593 & Alpine & 53.6 & 5.97 & 16.7 & 4.41 & 16.4 \\
\hline 6617 & Alpine & 41.4 & 2.83 & 6.1 & 1.18 & 6.29 \\
\hline 6618 & Alpine & 60.6 & 5.07 & 7.45 & 2.07 & 8.87 \\
\hline 6621 & Alpine & 55.1 & 4.26 & 15.6 & 2.84 & 16.3 \\
\hline 6629 & Alpine & 40.7 & 2.17 & 9.38 & 1.42 & 10.4 \\
\hline 6630 & Alpine & 30.3 & 2.09 & 3.65 & 0.97 & 4.24 \\
\hline 6631 & Alpine & 51.5 & 5.12 & 16.3 & 3.43 & 15.9 \\
\hline 6632 & Alpine & 64.7 & 2.77 & 5.23 & 1.36 & 5.68 \\
\hline 6670 & Alpine & 52.8 & 2.95 & 4.97 & 2.06 & 6.66 \\
\hline 6673 & Alpine & 49.5 & 2.29 & 5.91 & 1.18 & 7.6 \\
\hline 6677 & Alpine & 53.2 & 3.05 & 2.86 & 2.18 & 4.16 \\
\hline 6678 & Alpine & 63.4 & 3.47 & 9.06 & 1.9 & 9.64 \\
\hline Average & Alpine & 51.55 & 3.75 & 8.89 & 2.101 & 9.553 \\
\hline 6616 & Anglo-Nubian & 17 & 0.78 & 3.61 & 0.72 & 4.15 \\
\hline 6622 & Anglo-Nubian & 41.2 & 4.12 & 15 & 3.04 & 17.7 \\
\hline 6623 & Anglo-Nubian & 31.8 & 2.56 & 23.9 & 2.04 & 25.2 \\
\hline 6625 & Anglo-Nubian & 36.3 & 3.22 & 16 & 2.57 & 17 \\
\hline 6679 & Anglo-Nubian & 52.6 & 0.67 & 3.62 & 0.64 & 4.1 \\
\hline 6682 & Anglo-Nubian & 62 & 1.84 & 6.51 & 1.65 & 6.85 \\
\hline 6686 & Anglo-Nubian & 36.4 & 2.23 & 11.6 & 1.89 & 13.2 \\
\hline Average & Anglo-Nubian & 39.61 & 2.2 & 11.46 & 1.793 & 12.6 \\
\hline 6620 & Saanen & 50.5 & 3.76 & 9.25 & 1.85 & 8.89 \\
\hline 6624 & Saanen & 9.59 & 0.53 & 0.53 & 0.34 & 0.95 \\
\hline 6671 & Saanen & 56.3 & 3.97 & 10.6 & 2.34 & 11.8 \\
\hline Average & Saanen & 38.8 & 2.75 & 6.79 & 1.51 & 7.213 \\
\hline 6619 & La Mancha & 32.9 & 4.64 & 19.1 & 5.56 & 21 \\
\hline 6627 & Toggenburg & 35.6 & 5.53 & 24.6 & 5.91 & 25.9 \\
\hline
\end{tabular}

Fig. 4. Flow cytometry plots of goat \#6593 (as one example for the gating strategy) at age 5.5 weeks. Left plot represents cell sorting according to cell size (FSC-A) and granulation (SSC-A). Monocytes are encircled as cells larger in size and low in granulation, while granulocytes were detected as cells with strong granulation and variable sizes. These cell populations were used for analysis for markers for MHC II (x-axis) and CD14 (y-axis) (middle plot). CD14+ cells were divided into cells with high amount of CD14 and those with lesser amounts (boxed accordingly). CD14 positive cells were then back-gated shown in the plot to the right (top box: CD14 high; bottom left box: CD14 low) and associated to their respective original cell population as monocytes and granulocytes, respectively. The table below the flow cytometry plots contains data for the various cell subpopulations associated with the two markers for each goat as well as the average of each breed

Table 3. Statistical analyses of potential breed differences of average cell amounts of peripheral $\mathrm{T}$ cell populations (F: F test statistic for one-way ANOVA, BH p-value represents Benjamini-Hochberg adjusted pvalue)

\begin{tabular}{lccclll}
\hline Cell type & Alpine & Anglo-Nubian & Saanen & F & P-value & BH p-value \\
\hline Cd4+ Panel 1 & 34.9640 & 31.0710 & 37.2000 & 0.728 & 0.495 & 0.809 \\
CD8+ & 13.9210 & 12.7170 & 13.0930 & 0.182 & 0.835 & 0.902 \\
CD4+/CD8+ & 0.4530 & 0.4950 & 0.5560 & 0.161 & 0.852 & 0.902 \\
CD4-/CD8-1 & 42.2860 & 49.0430 & 43.0000 & 0.755 & 0.482 & 0.809 \\
CD4+ Panel 2 & 43.3640 & 37.7430 & 43.3330 & 0.862 & 0.437 & 0.809 \\
WCI+ & 18.7860 & 18.9540 & 14.8000 & 0.336 & 0.718 & 0.902 \\
\hline
\end{tabular}



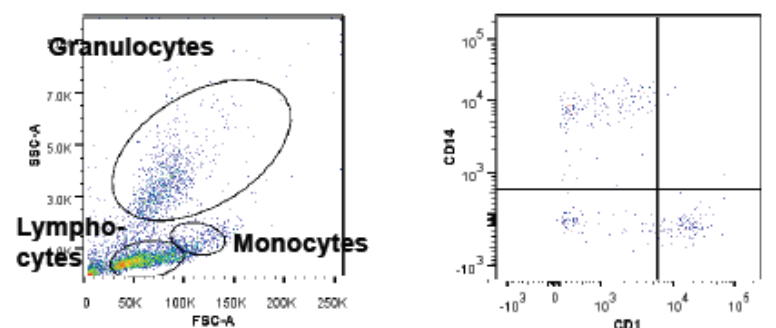

\begin{tabular}{|c|c|c|c|c|c|}
\hline Tag \# & Breed & CD14+ & CD14+/CD1+ & CD1+ & CD14-/CD1- \\
\hline 6578 & Alpine & 36.8 & 2.78 & 33 & 27.4 \\
\hline 6583 & Alpine & 17.8 & 2.11 & 61.4 & 18.7 \\
\hline 6593 & Alpine & 47.4 & 1.22 & 31 & 20.4 \\
\hline 6617 & Alpine & 24.7 & 0.77 & 44.4 & 30.1 \\
\hline 6618 & Alpine & 18 & 3.95 & 50 & 28.1 \\
\hline 6621 & Alpine & 31.7 & 1.93 & 39.4 & 27 \\
\hline 6629 & Alpine & 22 & 6.3 & 26 & 45.7 \\
\hline 6630 & Alpine & 13.4 & 2.68 & 60.4 & 23.5 \\
\hline 6631 & Alpine & 31.4 & 3.32 & 38.3 & 27 \\
\hline 6632 & Alpine & 19.9 & 0 & 43.4 & 36.8 \\
\hline 6670 & Alpine & 30.2 & 4.76 & 28.6 & 36.5 \\
\hline 6673 & Alpine & 23.3 & 0 & 34 & 42.7 \\
\hline 6677 & Alpine & 20.5 & 0 & 63.6 & 15.9 \\
\hline 6678 & Alpine & 26.8 & 2.59 & 38.3 & 32.3 \\
\hline Average & Alpine & 25.993 & 2.315 & 42.271 & 29.436 \\
\hline 6616 & Anglo-Nubian & 29.5 & 0 & 43.6 & 26.9 \\
\hline 6622 & Anglo-Nubian & 41.1 & 0.93 & 33.6 & 24.3 \\
\hline 6623 & Anglo-Nubian & 37.8 & 1.8 & 28.4 & 32 \\
\hline 6625 & Anglo-Nubian & 46.8 & 3.67 & 30.3 & 19.3 \\
\hline 6679 & Anglo-Nubian & 34.6 & 0 & 23.1 & 42.3 \\
\hline 6682 & Anglo-Nubian & 34.7 & 1.02 & 24.5 & 39.8 \\
\hline 6686 & Anglo-Nubian & 22.9 & 1.79 & 39.5 & 35.9 \\
\hline Average & Anglo-Nubian & 35.343 & 1.316 & 31.857 & 31.5 \\
\hline 6620 & Saanen & 21.6 & 2.95 & 53.4 & 22 \\
\hline 6624 & Saanen & 11.1 & 11.1 & 55.6 & 22.2 \\
\hline 6671 & Saanen & 25.2 & 1.26 & 35.5 & 38 \\
\hline Average & Saanen & 19.3 & 5.103 & 48.167 & 27.4 \\
\hline 6619 & LaMancha & 62.1 & 0.43 & 21.8 & 15.7 \\
\hline 6627 & Toggenburg & 62.4 & 0.95 & 17.9 & 18.8 \\
\hline
\end{tabular}

Fig. 5. Flow cytometry plots of goat \#6593 (as one example for the gating strategy) at age 5.5 weeks. Left plot represents cell sorting according to cell size (FSC-A) and granulation (SSC-A). Monocytes are encircled as cells larger in size and low in granulation. This cell population was used for analysis for markers for CD14 (y-axis) and CD1 (x-axis) (right plot). The table below the flow cytometry plots contains data for the various cell subpopulations associated with the two markers for each goat as well as the average of each breed

Table 4. Statistical analyses of potential breed differences of average amounts of peripheral CD14 bearing cell populations (F: F test statistic for one-way ANOVA, BH p-value represents Benjamini-Hochberg adjusted p-value)

\begin{tabular}{lllllll}
\hline Cell type & Alpine & Anglo-Nubian & Saanen & F & P-value & BH p-value \\
\hline CD14+ Monocytes & 2.101 & 1.793 & 1.510 & 0.601 & 0.557 & 0.836 \\
CD14+ Granulocytes & 9.553 & 12.60 & 7.213 & 1.130 & 0.342 & 0.809 \\
\hline
\end{tabular}


Darcy M. Fletcher et al. / American Journal of Animal and Veterinary Sciences 8 (4): 177-189, 2013

Table 5. Statistical analyses of potential breed differences of average amounts of peripheral antigenpresenting cell populations (F: $F$ test statistic for one-way ANOVA, BH p-value represents Benjamini-Hochberg adjusted p-value)

\begin{tabular}{|c|c|c|c|c|c|c|}
\hline Cell type & Alpine & Anglo-Nubian & Saanen & $\mathrm{F}$ & P-value & BH p-value \\
\hline CD14+ Panel 1 & 25.9930 & 35.3430 & 19.3000 & 4.610 & 0.022 & 0.197 \\
\hline CD1+ & 42.2710 & 31.8570 & 48.1670 & 3.002 & 0.071 & 0.358 \\
\hline $\mathrm{CD} 14+/ \mathrm{CD} 1+$ & 2.3150 & 1.3160 & 5.1030 & 2.860 & 0.080 & 0.358 \\
\hline CD14-/CD1- & 29.4360 & 31.5000 & 27.4000 & 0.261 & 0.773 & 0.902 \\
\hline CD14+ Panel 2 & 8.7210 & 13.4770 & 5.9870 & 6.671 & 0.006 & 0.102 \\
\hline MHC II+ & 54.2570 & 44.8860 & 57.4000 & 2.420 & 0.113 & 0.408 \\
\hline CD14+/MHC II+ & 18.5360 & 22.5280 & 17.7670 & 0.840 & 0.446 & 0.800 \\
\hline CD14-/MHC II- & 18.4830 & 19.1000 & 18.8670 & 0.017 & 0.983 & 0.983 \\
\hline
\end{tabular}
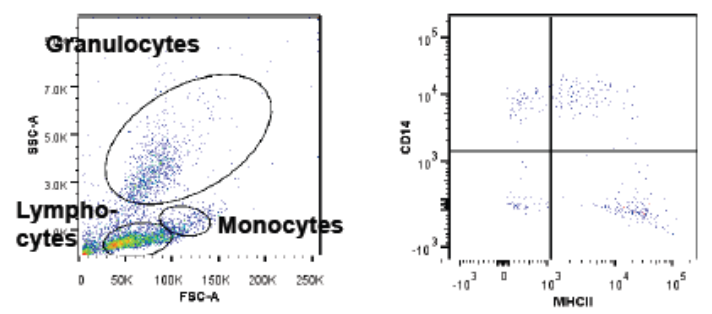

\begin{tabular}{|c|c|c|c|c|c|}
\hline Tag \# & Breed & CD14+ & CD14+/MHC II + & MHC II+ & CD14-/MHC II- \\
\hline 6578 & Alpine & 6.94 & 31.6 & 42.7 & 18.8 \\
\hline 6583 & Alpine & 10.8 & 7.77 & 70.4 & 11.1 \\
\hline 6593 & Alpine & 16.4 & 30.4 & 40.7 & 12.5 \\
\hline 6617 & Alpine & 5.79 & 19.3 & 57.5 & 17.4 \\
\hline 6618 & Alpine & 5.26 & 15.4 & 65.8 & 13.6 \\
\hline 6621 & Alpine & 11.6 & 22 & 52.1 & 14.3 \\
\hline 6629 & Alpine & 7.87 & 17.3 & 33.9 & 40.9 \\
\hline 6630 & Alpine & 8.72 & 6.04 & 67.1 & 18.1 \\
\hline 6631 & Alpine & 8.59 & 25.6 & 50 & 15.8 \\
\hline 6632 & Alpine & 5.15 & 14.7 & 58.8 & 21.3 \\
\hline 6670 & Alpine & 11.1 & 23.8 & 44.4 & 20.6 \\
\hline 6673 & Alpine & 5.83 & 15.5 & 57.3 & 21.4 \\
\hline 6677 & Alpine & 7.95 & 12.5 & 69.9 & 9.66 \\
\hline 6678 & Alpine & 10.1 & 17.6 & 49 & 23.3 \\
\hline Average & Alpine & 8.721 & 18.536 & 54.257 & 18.483 \\
\hline 6616 & Anglo-Nubian & 7.69 & 21.8 & 55.1 & 15.4 \\
\hline 6622 & Anglo-Nubian & 9.35 & 31.8 & 47.7 & 11.2 \\
\hline 6623 & Anglo-Nubian & 17.6 & 21.6 & 41.4 & 19.4 \\
\hline 6625 & Anglo-Nubian & 15.6 & 33 & 38.5 & 12.8 \\
\hline 6679 & Anglo-Nubian & 15.4 & 19.2 & 46.2 & 19.2 \\
\hline 6682 & Anglo-Nubian & 18.4 & 17.3 & 33.7 & 30.6 \\
\hline 6686 & Anglo-Nubian & 10.3 & 13 & 51.6 & 25.1 \\
\hline Average & Anglo-Nubian & 13.477 & 22.528 & 44.886 & 19.1 \\
\hline 6620 & Saanen & 7.21 & 16.4 & 62.3 & 14.1 \\
\hline 6624 & Saanen & 3.7 & 18.5 & 63 & 14.8 \\
\hline 6671 & Saanen & 7.05 & 18.4 & 46.9 & 27.7 \\
\hline Average & Saanen & 5.987 & 17.767 & 57.4 & 18.867 \\
\hline 6619 & LaMancha & 20.5 & 41.2 & 31 & 7.33 \\
\hline 6627 & Toggenburg & 40.2 & 21.7 & 24.5 & 13.6 \\
\hline
\end{tabular}

Fig. 6. Flow cytometry plots of goat \#6593 (as one example for the gating strategy) at age 5.5 weeks. Left plot represents cell sorting according to cell size (FSC-A) and granulation (SSC-A). Monocytes are encircled as cells larger in size and low in granulation. This cell population was used for analysis for markers for CD14 (y-axis) and MHC II (x-axis) (right plot). The table below the flow cytometry plots contains data for the various cell subpopulations associates with the two markers for each goat as well as the average of each breed 
Figure 5 shows the cells bearing CD1 and/or CD14, while Fig. 6 presents cells bearing MHC class II and/or CD14 molecules. These cells were characterized by their cell surface expression of CD14, CD1 and MHC class II molecules. Although no statistical significances were found for any cell type after correcting for multiple testing, CD14+ Panel 1 and Panel 2 both have unadjusted p-values $<0.05$ (Table 5). For CD14+ from both panels Anglo Nubian goats had higher means compared to Alpine and Saanen goats.

\section{DISCUSSION}

Goats are excellent animal models for chronic infectious diseases, such as mycobacterial diseases (Johne's disease, bovine and human tuberculosis), melioidosis, or brucellosis (Soffler et al., 2012; KahlMcDonagh et al., 2006; Stewart et al., 2006; Bezos et al., 2010; Perez et al., 2011). Unfortunately, the goat model is rarely used due to lack of goat-specific antibodies, information on cross-reactivities of bovine, ovine, murine and human antibodies and baseline data on (1) peripheral leukocyte population determined by flow cytometry, (2) potential breed differences and (3) available conjugated antibodies for cell surface markers of various peripheral blood cell populations. For any study using the goat model, it is important to know potential differences between breeds if single breeds are not available. In our case, we received 26 goat kids of various breeds. Most of them were Alpine, Saanen and Anglo-Nubian, however, we also received one LaMancha and one Toggenburg. The main question was if differences in cell surface markers are present between the three major breeds investigated.

The analyses were performed for peripheral blood leukocytes at the age of 3.5 weeks (emphasis on lymphoid cells) and 5.5 weeks (emphasis on myeloid cells). There was a clear pattern that distinguished the various cell populations from each other. While granulocytes were classified as high in granulation, lymphocytes and monocytes were detected-as expectedas low in granulation. Lymphocytes and monocytes could be clearly separated by cell size.

While we analyzed cell populations as early as 3.5 weeks, Stabel and Robbe-Austerman (2011) describedtheir analyses of calf PBMCs at an earlier age (1 week) without any difficulties. Somvanshi et al. (1987) described the development and changes of the whole hematology of Cashmere goats at different ages from less than one month to 10 years. While those are primarily fiber goats, they found slightly different data for lymphocytes $(52.63 \%$ for male and $57 \%$ for female) and monocytes $(4.81 \%$ for male and $4.02 \%$ for female) compared to our dairy goat breed data with lymphocytes ranging from 41.58 to $57.02 \%$ and monocytes ranging from 1.42 to $4.23 \%$. Clearly, our numbers for granulocytes differ from those determined by Somvanshi et al. (1987). While our numbers are primarily in the single digits or teens, their numbers range between 38.69 and $42.56 \%$. Different breeds, higher elevation, or different methods could have attributed to varying granulocyte amounts. While they discussed their different data for erythrocytes as compared to European goats, type of breed and different nutrition were mentioned as plausible causes for those differences. Unfortunately, these seem to be the only intensive descriptions of early white cell populations and immune markers in goat kids.

Most of the cell population analyzed for the two different time points did not exhibit any significant differences due to time points or breeds. This is to our knowledge the first report on such differences. Goat kids start their lives with high numbers of lymphocytes and lower numbers of granulocytes and the number of granulocytes increases throughout the first months of life (Mbassa and Poulsen, 1991). Indeed, we see those changes in the granulocyte populations between the weeks of 3.5 and 5.5 of age. However, lymphocytes are still the major cell population within the PBMCs of goats (Eiselt et al., 2011). While the main purpose of this study was to determine potential breed difference, we think that this was noteworthy.

While one could assume that there might be breedspecific differences in lymphocytes, granulocytes and/or monocytes, none have been reported yet. Breed-specific leukocyte differences were detected for three cattle breeds (Friesian, Red Danish, Jersey cattle) with statistical significance (Flensburg and Willeberg, 1976). Also, MHC class I reactivity to various antigens differs significantly between breeds of cattle in Australia (Stear et al., 1987). None were reported so far for the three major dairy goat breeds used in this study.

In our study, we used several antibodies that crossreacted with the caprine system. The reactivities of these antibodies were against bovine (three), ovine (three) and human (one); however, all of the tested antibodies demonstrated strong cross-reactivity with the caprine system. Interestingly, Dagleish et al. (2012) described crossreactivities of cell surface marker antibodies for the ovine system with the cervine system (European Red Deer) (CD3, CD4, CD8, MHC II, $\gamma \delta$ TCR, CD21, CD79acy). This seems logical since ruminants are 
closely relatedspecies; however, we did not see any useful cross-reactivity for the highly species-specific lymphocyte marker CD3. Neither the bovine nor the ovine antibodies cross-reacted. Thus, it seems essential to generate such antibody for further studies. In an earlier study, Naessens et al. (1993) analyzed 189 antibodies for cell surface markers towards their potential of cross reactivity with domestic and wild ruminant species including cows, sheep, goats, buffalo and waterbuck. Monoclonal antibodies for CD3 demonstrated crossreactivity for the bovine system as well as for the buffalo and waterbuck systems but not for the ovine or caprine system. Of the markers of key interest for studies on the immune system of goats CD1w3, CD2, CD4, CD5, CD6, CD8, CD11a, CD11b, CD11c, CD18, CD25, WC1 and CD44 demonstrated strong cross-reactivity with the caprine system. Although the clones for those specific antibodies are not commercially available we can confirm that the commercially available antibodies used in our study have similar cross-reactivities as described by Naessens et al. (1993). Furthermore, while several follow-up studies analyzed newly designed antibodies, they were only tested for the bovine system and not the ovine or caprine system (Sopp and Howard, 1997; Sopp et al., 2001; 2007). The most comprehensive analyses of antibody crossreactivities were performed by the laboratory of Dr. Davis, in which most bovine antibodies currently in use were generated (Mosaad et al., 2006). Their intensive analysis confirmed many cross-reactivities but also ensured the lack of a specific antibody for CD3 marker in goat that could be used in caprine studies.

While the main purpose in this study was to obtain baseline profiles for peripheral blood leukocytes and to define potential differences in granulocytes, lymphocytes and monocytes due to breeds, we also analyzed the lymphocyte subpopulations of goat kids. There are only few published studies targeting subpopulation analyses at an early age in goats. Navarro et al. (1996) studied four seven-month old Murciano-Granadina goats (dairy goats breed) for the presence of CD2, CD4, CD8, MHC I, MHC II, WC1 and CD25 in peripheral blood, lymph nodes, spleen and ileal Peyer's patches. No statistical analyses were performed due to the small number of animals involved.

Since the presence of CD4+ and CD8 + T cells is well documented in various analyses, CD4/CD8 double positive cells are mostly seen as undifferentiated $\mathrm{T}$ cells, which later will become either CD4+ or CD8+. Although in the past the importance of CD4/CD8 double negative $\mathrm{T}$ cells was dubious, over the last decade, it was shown that they might play a key role in local defense systems in the intestinal system with their own stimulation through the MR1 bearing antigen-presenting cells. This newly identified immune defense cascade seems to eliminate bacterial and viral pathogens during early infection. Interestingly, in HIV infected people, these cells become exhausted over time, marking disease progression. This observation might be important for various chronic infectious diseases thathave their initial start in the intestinal system. Thus, it seems even more logical to analyze potential differences of CD4/CD8 double negative cells in various breeds. No statistically significant differences in CD4/CD8 double negative cell populations were observed between the three breeds.

Although data cannot be compared due to the age differences of the animals it is clear that our goat kids had higher $\mathrm{CD} 4+$ and $\mathrm{CD} 4+/ \mathrm{CD} 8+$, as well as lower CD8+ number compared to those determined by Navarro et al. (1996). Although different antibodies were used in our study and in the study by Navarro et al. (1996), it seems that the amount of $\gamma \delta \mathrm{T}$ cells are similar throughout the first seven months. The same group published a follow-up study on the postnatal evolution of lymphocyte subpopulations in peripheral blood of goats (Caro et al., 1998). They analyzed the same goat breed at various time points throughout the first seven months for the same markers. Interestingly, their numbers strongly vary from our data even for 1 month of age, suggesting differences between the goat breeds involved. The final study on subpopulations of lymphocytes was performed by Winnicka et al. (1999) on 18 Saanen breed goats from 1 day of age to 1.5 years of age. No specific numbers for $\mathrm{CD} 4+, \mathrm{CD} 8+$ and $\mathrm{CD} 4+/ \mathrm{CD} 8+$ cells were provided during their discussion. Although they did not analyze lymphocyte subpopulations at 1 month, it seems when analyzing Fig. 3 in their study, however, that the CD8+ cell amount is similar to the number in our study. This last study on Saanen goats used a statistically significant amount of goats while the other two studies used only four animals. In comparison, our study included 14 goats of the Alpine breed, six Saanen goats and only four Anglo-Nubian goats.

\section{CONCLUSION}

While the study has its limitations by the number of goat kids per breed involved in the analyses and by the inclusion of only male goat kids, it still provides excellent baseline data on peripheral blood leukocytes and profiles of various $\mathrm{T}$ cell population and antigen- 
presenting cell surface maker population profiles. From our statistical analyses, two additional conclusions could be made: (1) any goat study could include goats from the three dairy goat breeds used in our analyses (Alpine, Anglo-Nubian, Saanen) and (2) many antibodies for various $\mathrm{T}$ cells and cell surface markers generated for cows, sheep, or humans could be successfully used in the goat model.

\section{ACKNOWLEDGEMENT}

This project was supported by Agriculture and Food Research Initiative competitive grant no. 2012-6701519379 from the USDA National Institute of Food and Agriculture to Torsten M. Eckstein.

\section{REFERENCES}

Benjamini, Y.and Y. Hochberg, 1995. Controlling the false discovery rate: A practical and powerful approach to multiple testing. J. Royal Statist. Soc. B, 57: 289-300.

Bezos, J., L. de Juan, B. Romero, J. Alvarez and F. Mazzucchelli et al., 2010. Experimental infection with Mycobacterium caprae in goats and evaluation of immunological status in tuberculosis and paratuberculosis co-infected animals. Vet. Immunol. Immunopathol., 133: 269-275. DOI: 10.1016/j.rvsc.2011.05.020

Caro, M.R., M.C. Gallego, A.J. Buendia, E. Navarro and J.A. Navarro, 1998. Postnatal evolution of lymphocyte subpopulations in peripheral blood and lymphoid organs in the goat. Res. Vet. Sci., 65: 145148. PMID: 9839893

Dagleish, M.P., J. Finlayson, P.J. Steele, Y. Pang and S. Hamilton et al., 2012. Immunophenotype of cells within cervine rectoanal mucosa-associated lymphoid tissue and mesenteric lymph nodes. J. Comparat. Pathol., 146: 365-371. DOI: 10.1016/j.jcpa.2011.08.007

Dharmadhikari, A.S. and E.A. Nardell, 2008. What animal models teach humans about tuberculosis. Am. J. Respir Cell Mol. Biol., 39: 503-508. DOI: 10.1165/rcmb.2008-0154TR

Drescher, K.M. and D. Sosnowska, 2008. Being a mouse in a man's world: what TMEV has taught us about humandisease. Front Biosci., 13: 3775-3785. PMID: 18508472
Eiselt, N., H. Wagner and A. Wehrend, 2011. Haematological parameters in goats. Tierarztliche Praxis Grosstiere, 39: 49-55. PMID: 22138746

Flensburg, J. and P. Willeberg, 1976. Studies on age specific leukocyte and lymphocyte counts in normal Friesian, Red Danish and Jersey cattle in Denmark. Nord. Vet. Med., 28: 150-9. PMID: 1272756

Groschup, M.H. and A. Buschmann, 2008. Rodent models for prion diseases. Vet. Res., 39: 32-40. DOI: 10.1051/vetres:2008008

Hibiya, K., M. Furugen, F. Higa, M. Tateyama and J. Fujita, 2011. Pigs as an experimental model for systemic Mycobacterium avium infectious disease. Comp. Immunol. Microbiol. Infect. Dis., 34: 455464. DOI: 10.1016/j.cimid.2011.09.001

Horvat, B., 2009. Animal models for the study of emerging zoonotic viruses: Nipah and Hendra. Vet. J., 181: 207-208. DOI: 10.1016/j.tvj1.2008.12.009

Hviid, L., C.R. Marinho, T. Staalsoe and C. PenhaGonçalves, 2010. Of mice and women: Rodent models of placental malaria. Trends Parasitol., 26: 412-419. DOI: 10.1016/j.pt.2010.04.010

Kahl-McDonagh, M.M., P.H. Elzer, S.D. Hagius, J.V. Walker and Q.L. Perry et al., 2006. Evaluation of novel Brucella melitensis unmarked deletion mutants for safety and efficacy in the goat model of brucellosis. Vaccine, 24: 5169-5177. PMID: 16697090

Kahn, R.E., I. Morozov, H. Feldmann and J.A. Richt, 2012. 6th International Conference on Emerging Zoonoses. Zoonoses Public Health, 59: 2-31. DOI: 10.1111/j.1863-2378.2012.01539.x

Mbassa, G.K. and J.S. Poulsen, 1991. Leukocyte profile in growing dwarf and landrace kids. Zentralblatt Veterinarmedizin A, 38: 389-397. PMID: 1910241

McConnell, M.J., L. Actis and J. Pachon, 2013. Acinetobacter baumannii: Human infections, factors contributing to pathogenesis and animal models. FEMS Microbiol. Rev., 3: 130-155. DOI: 10.1111/j.1574-6976.2012.00344.x

Mosaad, A.A., A.R. Elbagory, A.M. Khalid, W.R. Waters and A. Tibary et al., 2006. Identification of monoclonal antibody reagents for use in the study of the immune response to infectious agents in camel and water buffalo. J. Camel Pract. Res., 13: 91-101.

Munson, E., D.T. Nardelli, B.K. Du Chateau, S.M. Callister and R.F. Schell, 2012. Hamster and murine models of severe destructive Lyme arthritis. Clin. Dev. Immunol. DOI: 10.1155/2012/504215 
Naessens, J., R.O. Olubayo, W.C. Davis and J. Hopkins, 1993. Cross-reactivity of workshop antibodies with cells from domestic and wild ruminants. Vet. Immunol. Immunopathol., 39: 283-290. PMID: 8310653

Navarro, J.A., M.R. Caro, J. Seva, M.C. Rosillo and M.A. Gomez et al., 1996. Study of lymphocyte subpopulations in peripheral blood and secondary lymphoid organs in the goat using monoclonal antibodies to surface markers of bovine lymphocytes. Vet. Immunol. Immunopathol., 51: 147-156. PMID: 8797284

Padilla-Carlin, D.J., D.N. McMurray and A.J. Hickey, 2008. The guinea pig as a model of infectious diseases. Comput. Med., 58: 324-340. PMID: 18724774

Perez, B.D.V., S. Lopez-Soria, M. Nofrarias, M. Martín and H.M. Vordermeier et al., 2011. Experimental model of tuberculosis in the domestic goat after endobronchial infection with Mycobacterium caprae. Clin. Vaccine Immunol., 18: 1872-1881. DOI: $10.1128 / C V I .05323-11$

Ross, T.M., N. Bhardwaj, S.J. Bissel, A.L. Hartman and D.R. Smith, 2012. Animal models of Rift Valley fever virus infection. Virus Res., 163: 417-423. DOI: 10.1016/j.virusres.2011.10.023

Shi, C., J. Shi and Z. Xu, 2011. A review of murine models of latent tuberculosis infection. Scand J. Infect. Dis., 43: 848-856. DOI: 10.3109/00365548.2011.603745.

Soffler, C., A.M. Bosco-Lauth, T.A. Aboellail, A.J. Marolf and R.A. Bowen, 2012. Development and characterization of a caprine aerosol infection model of melioidosis. PLoS One, 7: e43207-e43207. DOI: 10.1371/journal.pone.0043207

Somvanshi, R., J.C. Biswas, B. Sharma and G.L. Koul, 1987. Haematological studies on Indian pashmira goats. Res. Vet. Sci., 42: 124-126. PMID: 3823626

Sopp, P. and C.J. Howard, 1997. Cross-reactivity of monoclonal antibodies to defined human leukocyte differentiation antigens with bovine cells. Vet. Immunol. Immunopathol., 56: 11-25. PMID: 9220577

Sopp, P., D. Werling and C. Baldwin, 2007. Crossreactivity of mAbs to human $\mathrm{CD}$ antigens with cells from cattle. Vet. Immunol. Immunopathol., 119: 106-114. PMID: 17675249
Sopp, P., L.S. Kwong and C.J. Howard, 2001. Crossreactivity with bovine cells of monoclonal antibodiessubmitted to the 6th international workshop on human leukocyte differentiation antigens. Vet. Immunol. Immunopathol., 78: 197206. PMID: 11182157

Stabel, J.R. and S. Robbe-Austerman, 2011. Early immune markers associated with Mycobacterium avium subsp. paratuberculosis infection in a neonatal calf model. Clin. Vaccine Immunol., 18: 393-405. DOI: 10.1128/CVI.00359-10.

Stear, M.J., S.C. Brown, C.K. Dimmock, J.H. Dufty and D.J. Hetzel et al., 1987. Breed differences in the frequency of bovine lymphocyte antigens. Exp. Clin. Immunogenet., 4: 27-36. PMID: 3273412

Stewart, D.J., J.A. Vaughan, P.L. Stiles, P.J. Noske and M.L. Tizard et al., 2006. A long-term study in Angora goats experimentally infected with Mycobacterium avium subsp. paratuberculosis: Clinical disease, faecal culture and immunological studies. Vet. Microbiol., 113: 13-24. PMID: 16310981

Uzal, F.A. and B.A. McClane, 2012. Animal models to study the pathogenesis of enterotoxigenic Clostridium perfringens infections. Microbes Infect., 14: 1009-1016. DOI: 10.1016/j.micinf.2012.06.003

Williams, K.L., S. Zompi, P.R. Beatty and E. Harris, 2009. A mouse model for studying dengue virus pathogenesis and immune response. Ann. NY Acad. Sci., 1171: E12-E23. DOI: 10.1111/j.17496632.2009.05057.x

Winnicka, A., W. Klucinski, J. Kawiak, G. Hoser and Z. Ryniewicz et al., 1999. Lymphocyte subpopulations, null cells and MHC II positive cells in peripheral blood of goats at different ages. Small Ruminant Res., 33: 247-253. DOI: 10.1016/S09214488(99)00019-X

Yi, P. and L. Li, 2012. The germfree murine animal: An important animal model for research on the relationship between gut microbiota and the host. Vet. Microbiol., 157: 1-7. DOI: 10.1016/j.vetmic. 2011.10 .024

Zhang, S. and S.F. Moss, 2012. Rodent models of Helicobacter infection, inflammation and disease. Methods Mol. Biol., 921: 89-98. PMID: 23015495 